\title{
Transferrin and folic acid co-modified bufalin-loaded nanoliposomes: preparation, characterization, and application in anticancer activity
}

This article was published in the following Dove Press journal:

International Journal of Nanomedicine

\section{Qiankun Chen' \\ Ji Liu²}

'Department of Thoracic Surgery, Shanghai Pulmonary Hospital, Tongji University School of Medicine, Shanghai, China; ${ }^{2}$ Department of Anesthesia, Shanghai Pulmonary Hospital, Tongji University School of Medicine, Shanghai, China
Correspondence: Ji Liu

Department of Anesthesia, Shanghai Pulmonary Hospital, Tongji University School of Medicine, 507 Zheng Min Road, Yangpu, Shanghai 200433, China

Tel/fax +86 2I 65II 5006

Email liuji05I5@yeah.net
Aim: The aim of this study was to prepare transferrin (Tf) and folic acid (FA) co-modified bufalin (BF) liposomes for lung cancer treatment.

Method: In this study, (FA+Tf) BF-LPs were prepared using the high-pressure homogenization method.

Results: The EE\% and DL\% of prepared LPs were $82.3 \%$ and $10.7 \%$, respectively, and the mean diameter was $120.4 \mathrm{~nm}$ from three batches. In vitro release showed that the release of BF from (FA+Tf) BF-LPs was slow with burst effects at an early stage. In vitro cytotoxicity assay showed that (FA+Tf) BF-LPs had a superior antiproliferative effect on A549 cells. An in vivo imaging study indicated that (FA+Tf) BF-LPs had obvious targeting characteristics on subcutaneous tumor, with the potential to actively deliver drugs to tumor tissues. In terms of the in vivo antitumor activity, (FA+Tf) BF-LPs treated mice showed a significantly suppressed tumor growth and no systemic toxicity in the body.

Conclusion: Through this study, it was found that the Tf and FA co-modified BF could be a very promising lung target preparation.

Keywords: transferrin, folic acid, liposomes, bufalin, in vivo imaging, antitumor activity

\section{Introduction}

Lung cancer is the leading cause of cancer-related death among men worldwide. It is the second leading cause of cancer-related death in women, with the 5-year survival rate only being $18 \% .^{1}$ Lung cancers are classified into small-cell lung cancer $(13 \%)$ and non-small-cell lung cancer (NSCLC) (87\%) according to the therapeutic purpose. ${ }^{2}$ Surgical resection is still the main treatment for early stage NSCLC. Unfortunately, the majority of lung cancers are diagnosed at an advanced stage. For advanced NSCLC, chemotherapy is currently the standard first-line treatment. ${ }^{3}$ However, the response rate to chemotherapy is less than $30 \%$. What is worse, many patients suffer serious side effects after chemotherapy.

Bufalin (BF), a soluble digoxin-like immune active component of Chansu, is an extract from the skin and parotid venom glands of Bufo gargarizans or Bufo melanostictus..$^{47}$ It has a significant antitumor effect against various tumors, including lung cancer, ${ }^{8}$ hepatocellular carcinoma,${ }^{9}$ colorectal cancer,${ }^{10}$ leukemia, ${ }^{11}$ and gastric cancer. ${ }^{12}$ A large number of studies have shown that antitumor effects of BF include inhibiting cell proliferation and inducing tumor cell apoptosis. ${ }^{13-15}$ Because of its toxicity, insolubility in water, fast metabolism, and short half-life, ${ }^{16}$ its application 
in cancer treatment is limited. Thus, to further explore valuable and novel therapeutic strategies is in urgent need.

The nanoparticulate drug delivery system has made significant contributions to the improvement of cancer drug delivery. ${ }^{17}$ Targeted nanoparticulate drug delivery systems, especially biodegradable nanoparticles, provide an opportunity to meet these existing challenges, with improved pharmacokinetics, benefits to drug accumulation in tumor tissue, and reduced side-effects. ${ }^{18}$ Folate receptor (FR) combined with folic acid (FA) has high affinity and mediates its intracellular transport through receptor-mediated endocytosis. ${ }^{19}$ There are three isoforms of FR (FR $\alpha$, FR $\beta$, and FR $\gamma$ ), and type $\alpha$ is the main subtype of FA transport. It is overexpressed on the surface of various types of tumors, including pancreatic, prostate, lung, head and neck, breast and ovarian cancers, and mesothelioma. ${ }^{20,21}$ Based on its limited expression and distribution in normal tissue, FR $\alpha$ is the most widely studied member of the FR family, and various strategies for targeting FR, which is overexpressed in cancer, have been developed. ${ }^{22}$ Similarly, the transferrin (Tf) receptor has been widely used in drug delivery to cancer cells due to its overexpression in many types of cancer, which is the result of its role in iron homeostasis and cell proliferation, as well as its receptor-mediated endocytosis. ${ }^{23}$ Many chemotherapeutic agents (such as oxaliplatin, doxorubicin, 5-fluorouracil, ceramide, as well as DNA and siRNA) ${ }^{24}$ have been used to inhibit tumor by Tf-mediated liposomes (LPs).

With the further development of active targeting agents, the strategy of modifying two ligands on the one nanocarrier is becoming more and more popular. Inspired by the strategy adopted by the natural virus, it is possible to develop a combination of ligands that interact with a variety of cell surface antigens to overcome the heterogeneous expression patterns in various tumor types and to increase the number of potential binding sites for nanomedicines, both on the surface of and within tumor cells. ${ }^{25,26}$

However, very few techniques involving nanoliposomes coupled with FA and Tf dual-targeting have been described, to the best of our knowledge. In the present study, we developed nanosized and highly efficient targeted BF nanoliposomes modified with FA and Tf to verify the hypothesis that targeted nanoliposomes can enhance the effect of antitumors.

\section{Materials and methods \\ Materials}

BF was purchased from Pure-one Bio Technology, Co., Ltd. (Shanghai, China). 1,2-Distearoyl-sn-Glycero-3Phosphoethanolamine (DSPE), DSPE-PEG2000-FA, and DSPE-PEG2000-Tf were obtained from Ponsure Biopharma Co., Ltd. (Shanghai, China). Egg phosphatidylcholine (EPC) and cholesterol (CHOL) were obtained from Sinopharm Chemical Reagent (Shanghai, China). The A549 lung cancer cell line was provided by the Institute of Biochemistry and Cell Biology, Chinese Academy of Sciences (Shanghai, China). Methanol, ethanol, ammonium acetate, and ethyl acetate were purchased from Suzhou Chemical Reagent Factory (Suzhou, China) and were of at least analytical grade. Watsons' water was used in all experiments.

\section{Preparation of LPs}

FA and Tf co-modified BF nanoliposomes (FA+TfBF-LPs) were prepared using the high-pressure homogenization method (Figure 1A). In brief, BF, EPC, CHOL, DSPEPEG2000-FA, and DSPE-PEG2000-Tf (molar ratio: 2, $55,33,5$, and 5 , respectively) were dissolved in $6.5 \mathrm{~mL}$ of chloroform to form a mixed solution, then the organic solvent was removed under reduced pressure at $40^{\circ} \mathrm{C}$ by
A

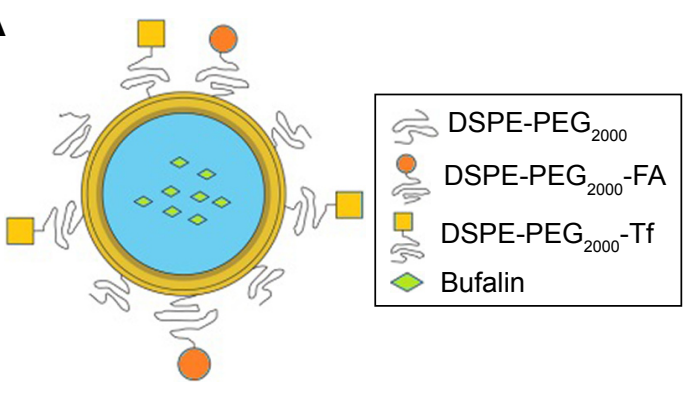

B

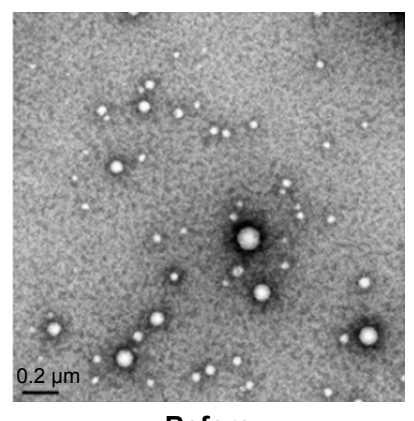

Before

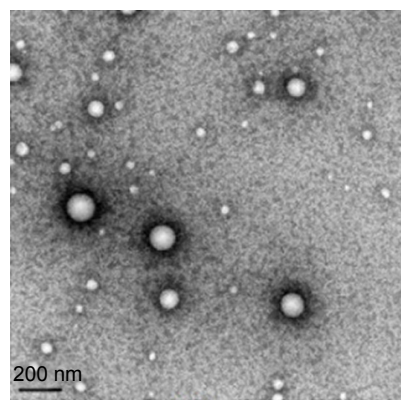

After

Figure I A schematic diagram of the structure of nanoliposomes (A); Transmission electron microscope of (FA+Tf) BF-LPs before and after storage (50,000X) (B). Abbreviations: FA, folic acid; Tf, transferrin; BF, bufalin; LP, liposome. 
rotary evaporation to form a thin film on the inner walls of the round-bottomed flask. The vacuum was applied for 1 hour to ensure total removal of any solvent trace. Glucose and mannitol $(1: 1, \mathrm{w} / \mathrm{w})$ were dissolved in phosphate buffer saline (PBS) $(\mathrm{pH}=7.4)$. The lipid film was then hydrated with $5 \mathrm{~mL}$ of $\mathrm{PBS}(\mathrm{pH}=7.4)$ at $55^{\circ} \mathrm{C}$. After that, the mixture was homogenized using a microfluidizer to obtain (FA+Tf) BF-LPs before the nanoliposomes were freeze-dried for 72 hours. Meanwhile, the PEGylated nanoliposomes (PEGLPs), FA-BF-LPs and Tf-BF-LPs, were also prepared with the above Materials and methods section.

\section{Characterization}

Particle morphology was investigated by transmission electron microscopy (TEM) after negative staining with phosphonic acid wolfram. The average particle size, zeta potential, and polydispersity index of BF LPs were determined by the laser light scattering method. BF was extracted from the LPs $(100 \mathrm{mg}$ ) with $2 \mathrm{~mL}$ acetonitrile, and then the extract solution was properly diluted prior to HPLC analysis. Encapsulation efficiency (EE\%) and drug loading (DL\%) were worked out by the following equations:

$$
\begin{gathered}
\mathrm{DL} \%=\frac{W_{\mathrm{M}}}{W_{\mathrm{P}}+W_{\mathrm{M}}} \times 100 \% \\
\mathrm{EE} \%=\frac{W_{\mathrm{M}}}{W_{\mathrm{F}}} \times 100 \%
\end{gathered}
$$

where $W_{\mathrm{P}}$ is the weight of initial feeding polymer, $W_{\mathrm{M}}$ is the weight of drug incorporated in LPs, and $W_{\mathrm{F}}$ is the weight of initial feeding drug.

\section{Stability}

The stability of freeze-dried (FA+Tf) BF-LPs was observed for 6 months at room temperature and $4{ }^{\circ} \mathrm{C}$ respectively. The LPs were observed mainly in regard to the following aspects: the average particle size, zeta potential, polydispersity index, EE\%, and DL\%. Each measurement was repeated three times.

\section{In vitro release}

The in vitro release properties of BF from different LPs were investigated in an aqueous medium containing PBS ( $\mathrm{pH}=7.4$ ), using the dialysis bag method. Different BF LPs were dispersed into $2 \mathrm{~mL}$ of release buffer in dialysis bags (Mol $\approx 10,000$ ), which were then put into a tube containing $200 \mathrm{~mL}$ of $\mathrm{pH}$ 7.4 PBS. All the samples were under the sink condition and kept in a constant shaking bath at $80 \mathrm{rpm}$ maintained at $37^{\circ} \mathrm{C}$. At time intervals $(0.25,0.5,1,2,4,6$, $8,10,24$, and 48 hours), $2 \mathrm{~mL}$ of dialysate was taken for analysis and then $2 \mathrm{~mL}$ of fresh medium was added. The concentration of $\mathrm{BF}$ in the release medium was determined by HPLC after filtration and proper dilution. At the same time, the release of free BF and unmodified BF LPs were monitored as a control.

\section{In vitro cytotoxicity assay}

The cytotoxicity of BF formulated in FA and Tf co-modified LPs was assessed in A549 cancer cell lines and compared to free BF and other LPs. Briefly, cells were seeded in 96-well plates followed by 24 hours of incubation in RPMI-1640 medium with $10 \%$ FBS and $1 \%$ streptomycin-penicillin. Twenty-four hours later, cells were incubated with a different sample with varying concentrations of free $\mathrm{BF}$, PEG-LPs, FA-BF-LPs, Tf-BF-LPs, and (FA+Tf) BF-LPs (from $0.002 \mu \mathrm{g} / \mathrm{mL}$ to $20 \mu \mathrm{g} / \mathrm{mL}$ ). Cells were incubated for 72 hours and cell viability was assessed by MTT (3-(4,5dimethyl-thiazol-2-yl)-2,5-diphenyltetrazolium bromide) assay in accordance with the following procedure: $1 \mathrm{~mL}$ of complete growth culture medium and $60 \mu \mathrm{L}$ of MTT solution $(5 \mathrm{mg} / \mathrm{mL}$ in PBS) were added to each well for 4 hours of incubation. The absorbance was measured with a microplate reading instrument at $540 \mathrm{~nm}$. The results were expressed as percentages relative to the results obtained with a non-toxic control. To determine whether this is due to FA- or Tf-mediated active targeting, free FA $(100 \mu \mathrm{M})$ and $\operatorname{Tf}(100 \mu \mathrm{M})$ were coadded to cells with FA-BF-LPs and Tf-BF-LPs, respectively.

\section{Cell uptake}

The cellular internalization of blank LPs, free BF, PEG BFLPs, FA-BF-LPs, Tf-BF-LPs, and (FA+Tf) BF-LPs was observed through confocal microscopy by using coumarin-6 as a fluorescent probe. A549 cells were grown in the RPMI 1640 medium, and 10\% (V/V) FBS and 5\% antibiotics were added in the medium (100 IU/mL penicillin G sodium and $100 \mu \mathrm{g} / \mathrm{mL}$ streptomycin). A549 cells were seeded in a cell culture dish with an initial density of $4 \times 10^{5}$ cells/dish. Cells were then incubated with coumarin-6-adsorbed blank LPs, free BF, PEG BF-LPs, FA-BF-LPs, Tf-BF-LPs, and (FA+Tf) BF-LPs for 2 hours at $37^{\circ} \mathrm{C} \pm 0.5^{\circ} \mathrm{C}$. Subsequently, cells were washed several times with PBS, and fixed with $4 \%$ paraformaldehyde for 10 minutes. Finally, cells were observed under a confocal microscope. For the quantitative estimation of BF uptake, the density of cells inoculated on 
24-well plates was $3 \times 10^{4}$. When they reached $70 \%-80 \%$ confluence, cells were incubated with coumarin-6-adsorbed blank LPs, free BF, PEG BF-LPs, FA-BF-LPs, Tf-BF-LPs, and (FA+Tf) BF-LPs. After 2 hours of culture, cells were washed several times with cold PBS. Subsequently, Triton $\mathrm{X}-100(0.1 \%)$ was added to dissolve the cells. Fluorescence intensities were measured by multi-mode microplate reader at an excitation wavelength of $440 \mathrm{~nm}$ and an emission wavelength of $520 \mathrm{~nm}$.

\section{In vivo studies}

\section{Animal}

All BALB/c nude mice (male, 18-20 g, 6-8 weeks) were provided by the Shanghai Experimental Animal Center of Chinese Academy of Sciences (Shanghai, China). All protocols were approved by the Committee on Animals of the Tongji University School of Medicine (Shanghai, China), and were carried out in accordance with the guidelines of the Committee on Animals of the Tongji University School of Medicine.

\section{In vivo imaging}

The noninvasive optical imaging systems were used to observe the real-time distribution of the functional targeting LPs in A549 cells of xenograft mice. DiR was encapsulated into LPs as a fluorescence probe. $1 \times 10^{9}$ A549 cells were injected into the right forelimb flank of mice according to the previous report, in order to build the tumor-bearing mice models. When the tumor volume reached $\sim 100-150 \mathrm{~mm}^{3}$, the mice were injected with blank LPs, FA-BF-LPs, Tf-BFLPs, and (FA+Tf) BF-LPs $(0.2 \mathrm{mg} / \mathrm{kg})$ via the tail vein (three each group). Prior to in vivo imaging, the mice were anesthetized with phenobarbital sodium. At 2, 8, and 24 hours, an in vivo imaging system was used to scan the images of animals. Fluorescence imaging was performed with an excitation wavelength of $490 \mathrm{~nm}$ and emission wavelength of $535 \mathrm{~nm}$. The exposure time was 1 minute.

\section{The antitumor activity}

Briefly, on day $0,1 \times 10^{9}$ A549 cells were injected SC into BALB/c nude mice. By day 14, the average tumor volume of each mouse reached about $100 \mathrm{~mm}^{3}$. From day 14, the mice were treated with different BF LPs or free BF at a dose of $15 \mathrm{mg} / \mathrm{kg}$ via the tail vein, and the drugs were given once every 3 days for 10 times. The tumor size and body weight were measured three times per week during the study. On day 50, the mice were euthanized, followed by excision and weighing of the tumors. The tumor volume (V) was calculated using the following formula: $\left(\mathrm{W}^{2} \times \mathrm{L}\right) / 2$, where $\mathrm{W}$ was the widest tumor measurement value and $\mathrm{L}$ was the longest tumor dimension. The relative tumor volume (R) was calculated by the following formula: Vi/V0, where V0 is the tumor volume on day 14 , and $\mathrm{Vi}$ is the tumor volume at the measurement point. Antitumor activity was analyzed by the relative tumor inhibitory rate $(\%)$, and the formula was as follows: (1-[R(treatment group)/R(negative control group)]) $\times 100 \%$.

\section{Statistical analysis}

The experimental data were expressed as mean and standard deviation (mean $\pm \mathrm{SD}$ ), and the significance of differences was analyzed using unpaired Student's $t$-test at $P<0.05$.

\section{Results and discussion Preparation and characterization}

Previous researchers reported on similar FA- and Tf-based LPs formulation for various other anticancer drugs. ${ }^{27-29}$ In this study, the FA and Tf co-modified BF LPs were prepared by the high-pressure homogenization method and the particle morphology was screened by TEM. As shown in Figure 1B, (FA+Tf) BF-LPs had a uniform spherical morphology. The mean particle size and polydispersity index of prepared (FA+Tf) BF-LPs were determined to be $120.4 \mathrm{~nm}$ and 0.121 , respectively. According to the literature, particle size is an important parameter as, although nanoparticles below the $400 \mathrm{~nm}$ size range can extravasate out of circulation into the tumor microspace, smaller sizes can internalize into the cell via endocytic vesicles more efficiently. ${ }^{30,31}$ Zeta potential of the reconstituted (FA+Tf) BF-LPs was determined to be $-16.8 \mathrm{mV}$. Quantitative analysis of EE\% and DL\% of (FA+Tf) BF-LPs and determination were finished by the RP-HPLC method. The EE\% was $82.3 \%$ and DL\% rate was $10.7 \%$ (Table 1). The zeta potentials of LPs were negative, due to the addition of DSPE-PEG2000. High EE\% and DL\% of drugs from the LPs would be beneficial to the prevention of the rapid leakage during the process of drug delivery and the accumulation of drug in tumor tissues.

\section{Stability}

The stability of the (FA+Tf) BF-LPs was important for storage. In our study, there were no significant differences in the average particle size, zeta potential, polydispersity index, $\mathrm{EE} \%$, and DL\% in 0, 1, 3, and 6 months $(P>0.05)$ (Table 1$)$. At the same time, the surface morphology showed that the shape of (FA+Tf) BF-LPs did not change after 6 months (Figure 1B). 
Table I The characteristics and stabilities data of different BF formulations before and after storage at different temperatures ( $\mathrm{n}=3$ )

\begin{tabular}{l|l|l|l|l|l|l|l}
\hline Formulations & Temperature & $\begin{array}{l}\text { Time } \\
\text { (months) }\end{array}$ & $\begin{array}{l}\text { Particle } \\
\text { size } \\
(\mathbf{n m})\end{array}$ & $\begin{array}{l}\text { Zeta } \\
\text { potential } \\
(\mathbf{m V})\end{array}$ & $\begin{array}{l}\text { Encapsulation } \\
\text { efficiency }(\%)\end{array}$ & $\begin{array}{l}\text { Drug } \\
\text { loading (\%) }\end{array}$ & $\begin{array}{l}\text { Polydispersity } \\
\text { index }\end{array}$ \\
\hline PEG BF-LPs & Room temperature & 0 & $116.6 \pm 8.4$ & $-17.2 \pm 0.7$ & $81.9 \pm 6.2$ & $9.7 \pm 1.3$ & 0.114 \\
\hline FA BF-LPs & Room temperature & 0 & $118.2 \pm 7.1$ & $-18.8 \pm 0.6$ & $82.2 \pm 4.9$ & $10.4 \pm 1.2$ & 0.118 \\
\hline Tf BF-LPs & Room temperature & 0 & $119.7 \pm 6.3$ & $-19.3 \pm 0.4$ & $83.1 \pm 4.7$ & $10.5 \pm 1.3$ & 0.124 \\
\hline (FA+Tf) BF-LPs & Room temperature & 0 & $120.4 \pm 9.1$ & $-16.8 \pm 0.5$ & $82.3 \pm 5.3$ & $10.7 \pm 1.1$ & 0.121 \\
& & 1 & $120.8 \pm 8.5$ & $-17.2 \pm 0.7$ & $82.1 \pm 6.1$ & $10.5 \pm 1.3$ & 0.123 \\
& & 3 & $121.1 \pm 6.7$ & $-17.1 \pm 0.3$ & $81.7 \pm 6.7$ & $10.8 \pm 1.4$ & 0.119 \\
& 4 & 6 & $121.6 \pm 7.4$ & $-17.5 \pm 0.6$ & $80.8 \pm 4.6$ & $10.3 \pm 0.9$ & 0.120 \\
& & 1 & $120.9 \pm 6.6$ & $-16.9 \pm 0.3$ & $81.9 \pm 6.3$ & $10.5 \pm 1.2$ & 0.119 \\
& & 3 & $121.2 \pm 7.2$ & $-16.8 \pm 0.9$ & $81.5 \pm 7.2$ & $10.4 \pm 1.1$ & 0.123 \\
& & 6 & $121.9 \pm 5.7$ & $-16.9 \pm 0.4$ & $80.3 \pm 6.5$ & $10.3 \pm 1.3$ & 0.122 \\
\hline
\end{tabular}

Abbreviations: FA, folic acid; Tf, transferrin; BF, bufalin; LP, liposome.

\section{In vitro release}

The release profiles of LPs with different BF formulations at $37^{\circ} \mathrm{C}$ are shown in Figure 2. The release of free BF was also studied as a control. In vitro release data showed that free $\mathrm{BF}$ was released faster than BF LPs, and the release of free $\mathrm{BF}$ hit $90 \%$ after 4 hours. For $(\mathrm{FA}+\mathrm{Tf}$ ) modified $\mathrm{BF}$ LPs, a low BF burst release of $20 \%$ was noted at 1 hour. For unmodified LPs (PEG BF-LPs), the initial burst of drug release $(30 \%, 1$ hour $)$ was obvious, which was attributable to the rapid diffusion of free drugs adsorbed on the particles. Compared with the unmodified, the release rate of BF from LPs was slower, with an extended drug release rate of over 10 hours, after which a plateau was observed till 48 hours.
This might be attributed to the surface adsorption coating, which could impede the diffusion of encapsulated drugs in the nanoscale system. It is worth noting that there is no significant difference between the release of (FA+Tf) BF-LPs, FA-BF-LPs, and Tf-BF-LPs in vitro. This was probably because their space structures did not vary much. It possibly suggested that the total drug-release amount from the LPs depended on the drug solubility and the penetration from the core of LPs.

\section{In vitro cytotoxicity assay}

Figure 3 shows the antiproliferative effect of different BF preparations on A549 lung cancer cells. The cells were

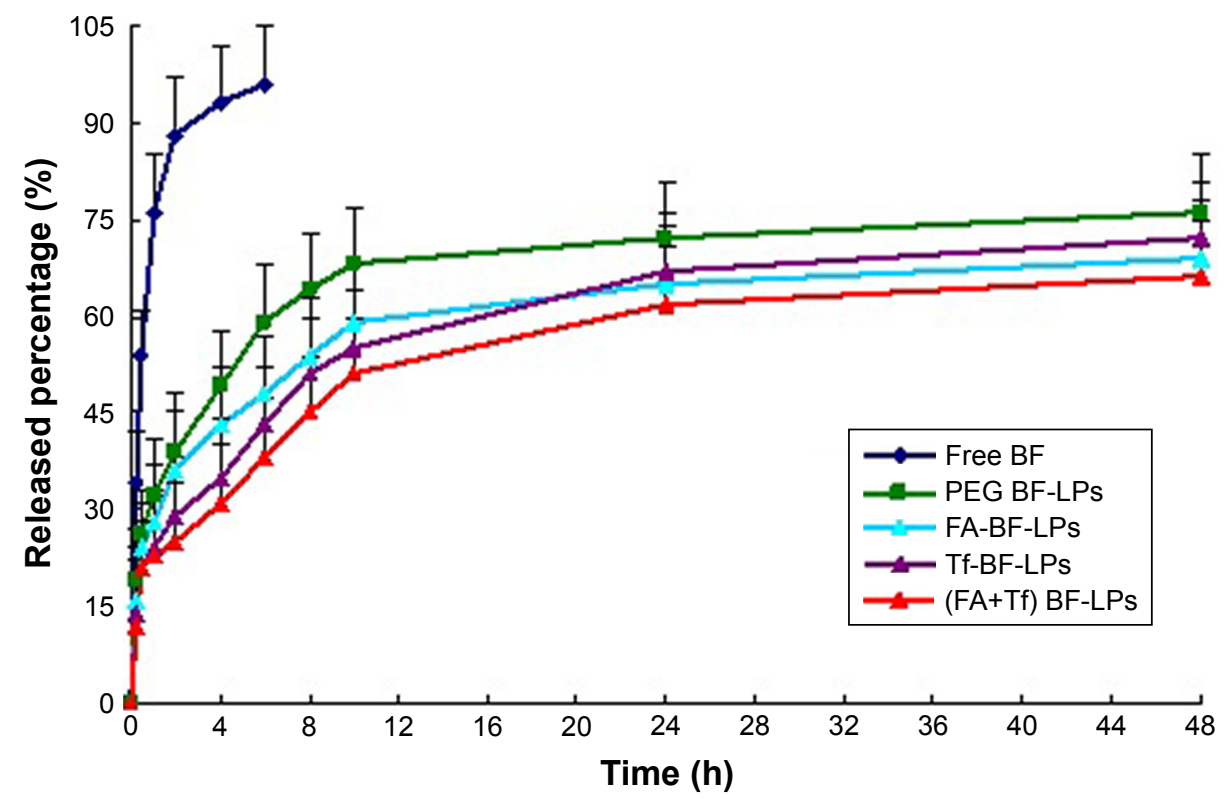

Figure 2 In vitro drug release profile of $B F$ from different formulations (PEG BF-LPs, FA-BF-LPs, Tf-BF-LPs, and (FA+Tf) BF-LPs) at pH 7.4 (data presented as mean \pm SD, $n=3$ ). Abbreviations: FA, folic acid; Tf, transferrin; BF, bufalin; LP, liposome; SD, standard deviation. 


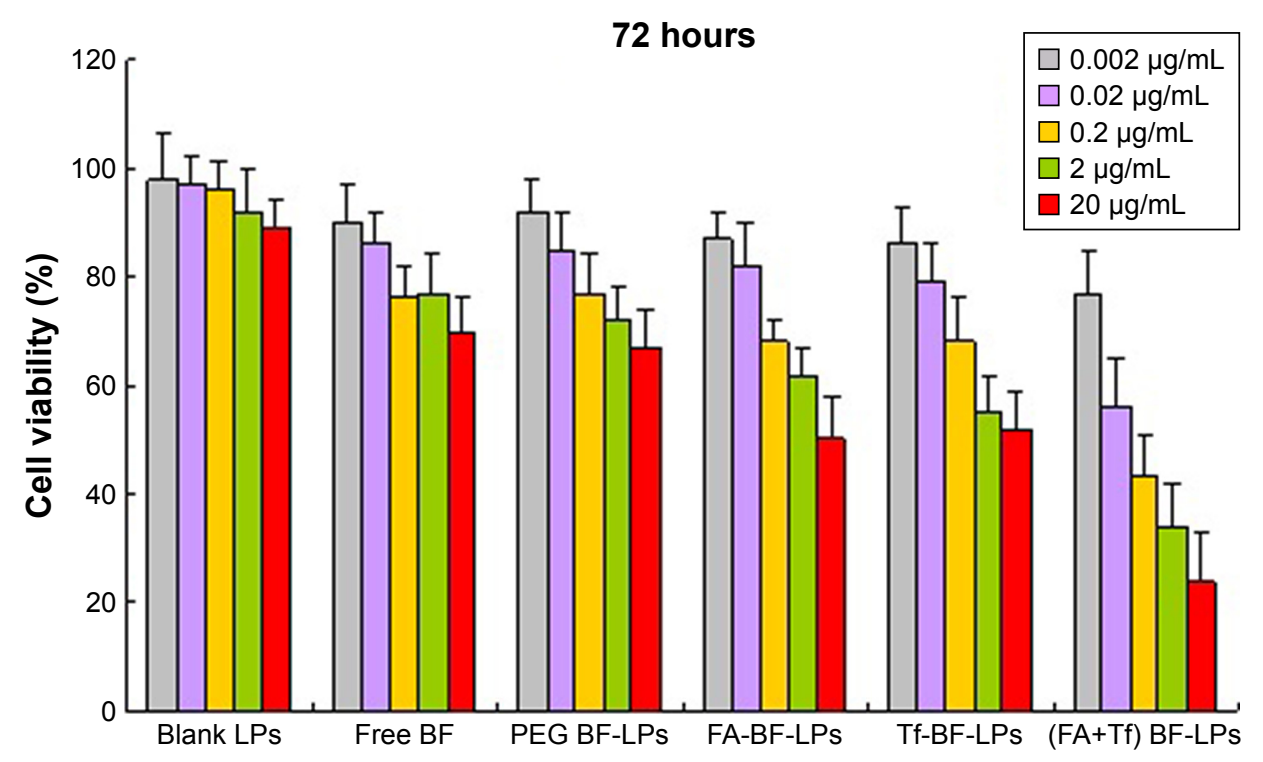

Figure 3 In vitro cytotoxicity analysis of blank LPs, free BF, PEG BF-LPs, FA-BF-LPs, Tf-BF-LPs, and (FA+Tf) BF-LPs on A549 cell lines. Notes: Cell viability assay was performed by MTT assay. Incubation time $=72$ hours. Abbreviations: FA, folic acid; Tf, transferrin; BF, bufalin; LP, liposome.

treated with different BF preparations, and the cytotoxicity was measured by MTT after 72 hours. All BF preparations showed time and concentration dependent cytotoxicity on A549 cells. As summarized in Table 2, The $\mathrm{IC}_{50}$ was 4.58 , $1.67,1.25,0.84,0.92$, and $0.44 \mu \mathrm{g} / \mathrm{mL}$ for blank LPs, free BF, PEG BF-LPs, FA-BF-LPs, Tf-BF-LPs, and (FA+Tf) BF-LPs, respectively. BF-loaded FA-Tf co-modified LPs were the most potent among all the BF formulations with respect to cell growth inhibition. It has been reported that $100 \mu \mathrm{M}$ free FA can block more than $99 \%$ of the binding by FA receptor. ${ }^{32}$ Indeed, the presence of excess free FA decreased the cytotoxicity of FA-BF-LPs, suggesting that the greater in vitro cytotoxicity of FA-BF-LPs could be attributed to the

Table $2 I_{50}$ values of BF different formulations in A549 cells following 72-hour treatments, respectively $(n=6)$

\begin{tabular}{l|l}
\hline Formulations & $\begin{array}{l}\text { A549 IC } \\
(\mu \mathrm{g} / \mathbf{m L})\end{array}$ \\
\hline Free BF & $62.6 \pm 5.4^{*}$ \\
PEG BF-LPs & $54.1 \pm 6.2^{*}$ \\
FA BF-LPs & $51.2 \pm 4.9^{*}$ \\
FA BF-LPs+FA (I00 $\mu \mathrm{M})$ & $59.7 \pm 4.8^{*}$ \\
Tf BF-LPs & $49.8 \pm 5.3^{*}$ \\
Tf BF-LPs+Tf (I00 $\mu \mathrm{M})$ & $55.4 \pm 6.5^{*}$ \\
(FA+Tf) BF-LPs & $21.4 \pm 2.7$ \\
(FA+Tf) BF-LPs+FA $(100 \mu \mathrm{M})+\mathrm{Tf}(100 \mu \mathrm{M})$ & $38.7 \pm 3.2^{*}$ \\
\hline
\end{tabular}

Note: $* P<0.05$ vs the group of (FA+Tf) BF-LPs.

Abbreviations: FA, folic acid; Tf, transferrin; BF, bufalin; LP, liposome. specific ligand-receptor interaction. The same phenomenon occurred in the group of Tf-BF-LPs.

\section{Cell uptake}

Since the coumarin-6-labeled different BF formulations were developed to trigger receptor-mediated internalization in tumor cells, we compared the uptake of the targeted group and the control group in A549 cell lines (Figure 4A). A strong green fluorescence was observed in the cytoplasmic region after the incubation of (FA+Tf) BF-LPs for 2 hours. Cell internalization in A549 cells was higher for FA and Tf co-modified BF LPs than others. On the contrary, in the control group, a few green fluorescent spots representing the blank NPs were observed on the cell membrane. The results showed that surface modification could reflect when the cell internalization process changed, and more drugs entered the cell successfully. In quantitative cell uptake studies, coumarin- 6 was quantified by recovering the drug LPs from cells and measuring their fluorescence on the five formulations (normalized to per $\mathrm{mg}$ of the total cellular protein contents). The quantitative results were consistent with the confocal images (Figure 4B).

\section{In vivo imaging study}

The in vivo tumor accumulation profiles of DiR-loaded BF LPs were clearly visualized by monitoring the whole body fluorescent intensity in subcutaneous xenograft bearing 
A
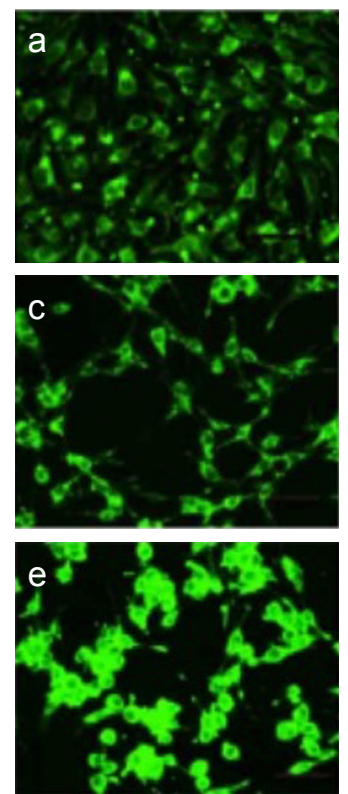
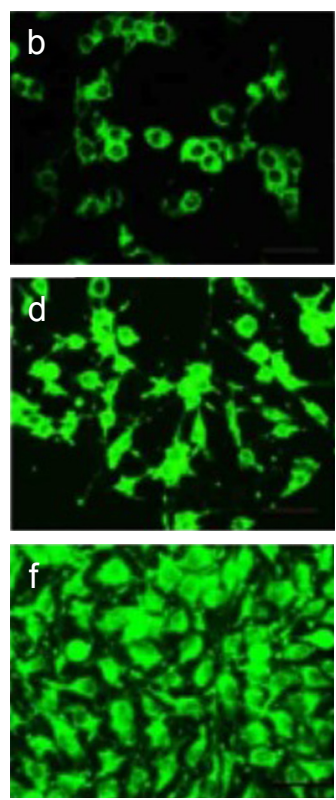

B
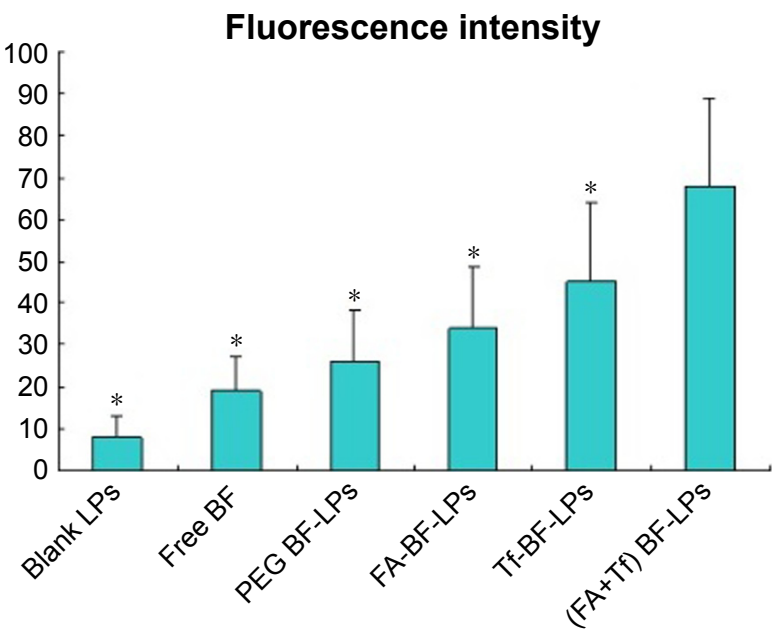

Figure 4 (A) Confocal images of cellular uptake of blank LPs (a), free BF (b), PEG BF-LPs (c), FA-BF-LPs (d), Tf-BF-LPs (e), and (FA+Tf) BF-LPs (f) by a 549 cells. Incubation time was 2 hours. (B) Mean fluorescence intensities for blank LPs, free BF, PEG BF-LPs, FA-BF-LPs, Tf-BF-LPs, and (FA+Tf) BF-LPs in quantitative cell uptake studies. *P $<0.05$ vs $(\mathrm{FA}+\mathrm{Tf}) \mathrm{BF}-\mathrm{LPs}$.

Abbreviations: FA, folic acid; Tf, transferrin; BF, bufalin; LP, liposome.

nude mice model (Figure 5). In the group of blank LPs, the fluorescence intensity gradually decreased in a short time. At 10 hours, the fluorescence intensity of tumor sites was almost invisible (the value of fluorescent intensity was close to 500). During the observation period, both FA-BF-LPs and Tf-BF-LPs showed certain fluorescence intensity. This indicated that the surface modification of LPs could play an active targeting function. However, the surprise appeared in the FA+Tf co-modified group, where the results showed that (FA+Tf) BF-LPs had stronger fluorescence in nude mouse subcutaneous tumor site than the other BF LPs at all times (the value of fluorescent intensity at the end of the observation period was close to 200-300). This phenomenon indicated that (FA+Tf) BF-LPs had obvious targeting characteristics on subcutaneous tumor, with a potential to actively deliver drugs to tumor tissues.

\section{The antitumor activity}

The tumor developed approximately 2 weeks following the subcutaneous injection of A549 cells, and the antitumor efficacy of blank LPs, free BF, PEG BF-LPs, FA-BF-LPs, Tf-BF-LPs, and (FA+Tf) BF-LPs was investigated in mice bearing A549 xenograft, when the tumor volume reached $\sim 100 \mathrm{~mm}^{3}$. During the experiment, the body weight and tumor volume were assessed every 3 days in order to evaluate the safety and efficacy of different treatments.

As illustrated in Figure 6A, the tumor showed an excessive growth in the control group treated with blank LPs and reached $653 \pm 112 \mathrm{~mm}^{3}$ in 32 days, while the tumor growth rate was inhibited remarkably in animals treated with free BF, PEG BF-LPs, FA-BF-LPs, Tf-BF-LPs, and (FA+Tf) BF-LPs. Also, there was a significant difference between the efficacy of (FA+Tf) BF-LPs and other treatments; in that, the minimal tumor volume was observed in the group treated with the (FA+Tf) BF-LPs delivery system $\left(94 \pm 23 \mathrm{~mm}^{3}\right)$, which was significantly less than in other groups treated with free BF, PEG BF-LPs, FA-BF-LPs, and Tf-BF-LPs. Figure 6B represents the changes of body weight in different groups during the 50-day study. During the entire period of the in vivo study, there were no significant body weight changes in all treatment groups compared with the blank LPs group. The average tumor weights and volumes are shown in Table 3. Compared with the control group, all treatment groups showed significantly $(P<0.05)$ inhibited tumor volume, with the greatest effect seen in the (FA+Tf) BF-LPs group. Similarly, both BF LPs could significantly inhibit the tumor weights. In the (FA+Tf) BF-LPs group, the effect was most obvious. Overall, our results indicated that 


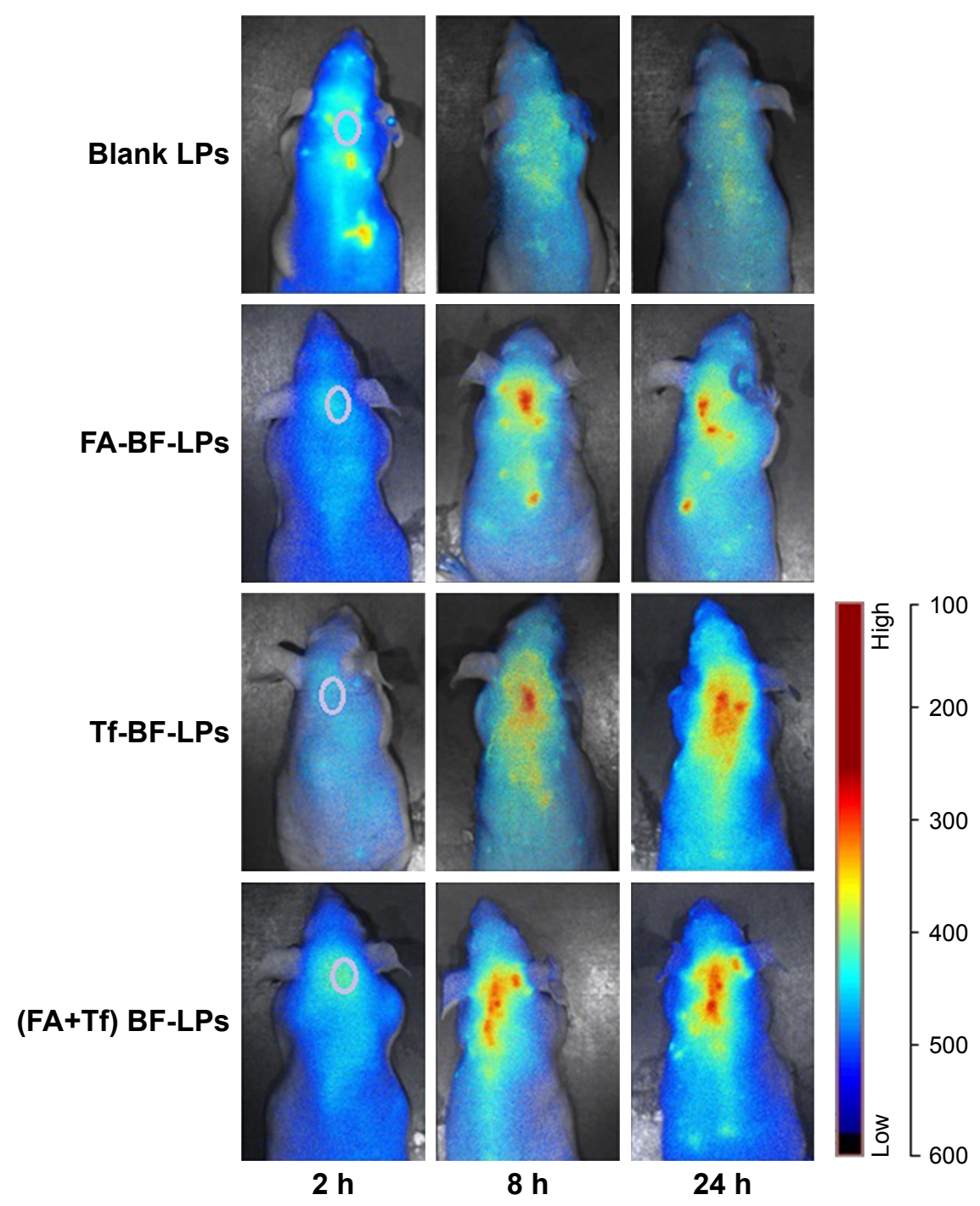

Figure 5 In vivo optical imaging of xenografts at different time intervals after administration of different DiR-labeled BF LPs. Note: The range from blue to red represents the changes in fluorescence intensity from weak to strong. Abbreviations: FA, folic acid; Tf, transferrin; BF, bufalin; LP, liposome.
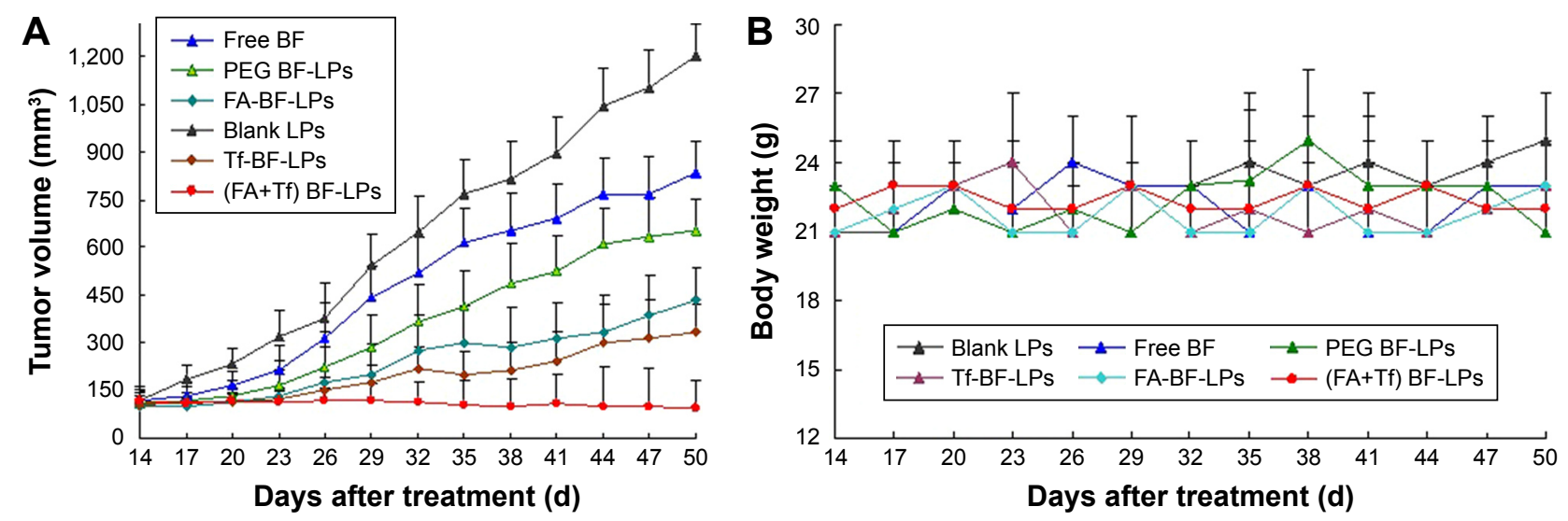

Figure 6 (A) A549 xenograft tumor growth inhibition by BF in different formulations. (B) Animal body weights. The body weights of treated animals were continuously monitored to investigate systemic cytotoxicity of BF in different formulations. Note: Data $=$ mean $\pm S D(n=8)$.

Abbreviations: FA, folic acid; Tf, transferrin; BF, bufalin; LP, liposome. 
Table 3 Effect of different BF LPs on A549 cells in nude mice

\begin{tabular}{|c|c|c|c|c|c|}
\hline \multirow[t]{2}{*}{ Formulation } & \multicolumn{2}{|c|}{$\operatorname{TV}\left(\mathrm{mm}^{3}\right)$} & \multirow[t]{2}{*}{ Tumor weight (g) } & \multirow[t]{2}{*}{$\mathbf{R}$} & \multirow[t]{2}{*}{ IR (\%) } \\
\hline & $d_{14}$ & $D_{50}$ & & & \\
\hline Blank LPs & $120 \pm 34$ & $\mathrm{I}, 202 \pm \mathrm{I} \mid 2$ & $0.875 \pm 0.121$ & $10.01 \pm 1.65$ & \\
\hline Free BF & $117 \pm 22$ & $832 \pm 101^{a}$ & $0.645 \pm 0.1 \mathrm{I}^{\mathrm{a}}$ & $7.11 \pm 1.13^{a}$ & 28.9 \\
\hline PEG-BF-LPs & $110 \pm 23$ & $654 \pm 67^{\mathrm{a}, \mathrm{b}}$ & $0.519 \pm 0.109^{\mathrm{a}, \mathrm{b}}$ & $5.95 \pm 1.16^{\mathrm{a}, \mathrm{b}}$ & 40.6 \\
\hline FA-BF-LPs & $109 \pm 19$ & $43 I \pm 43^{a, b, c}$ & $0.383 \pm 0.1 / 2^{a, b, c}$ & $3.95 \pm 0.98^{\mathrm{a}, \mathrm{b}, \mathrm{c}}$ & 60.5 \\
\hline Tf-BF-LPs & $105 \pm 26$ & $332 \pm 47^{\mathrm{a}, \mathrm{b}, \mathrm{c}}$ & $0.37 I \pm 0.104^{\mathrm{a}, \mathrm{b}, \mathrm{c}}$ & $3.16 \pm 0.78^{\mathrm{a}, \mathrm{b}, \mathrm{c}}$ & 68.4 \\
\hline$(F A+T f)-B F-L P s$ & $|20 \pm 3|$ & $94 \pm 23^{\mathrm{a}, \mathrm{b}, \mathrm{c}, \mathrm{d}, \mathrm{e}}$ & $0.20 I \pm 0.034^{\mathrm{a}, \mathrm{b}, \mathrm{c}, \mathrm{d}, \mathrm{e}}$ & $0.78 \pm 0.19^{\mathrm{a}, \mathrm{b}, \mathrm{c}, \mathrm{d}, \mathrm{e}}$ & 92.2 \\
\hline
\end{tabular}

Notes: ${ }^{a} P<0.05$, compared with blank LPs; ${ }^{b} P<0.05$, compared with free $B F ;{ }^{c} P<0.05$, compared with $P E G-B F-L P s$; ${ }^{d P}<0.05$, compared with FA-BF-LPs; ${ }^{e} P<0.05$, compared with Tf-BF-LPs.

Abbreviations: FA, folic acid; Tf, transferrin; BF, bufalin; LP, liposome; R, relative tumor volume; TV, tumor volume.

bifunctionally modified BF-LPs had a better antitumor effect in vivo than the single modified BF LPs.

\section{Disclosure}

The authors report no conflicts of interest in this work.

\section{References}

1. DeSantis CE, Lin CC, Mariotto AB, et al. Cancer treatment and survivorship statistics, 2014. CA Cancer J Clin. 2014;64(4):252-271.

2. Siegel R, Ma J, Zou Z, Jemal A. Cancer statistics, 2014. CA Cancer J Clin. 2014;64(1):9-29.

3. Zarogoulidis P, Pataka A, Terzi E, et al. Intensive care unit and lung cancer: when should we intubate? J Thorac Dis. 2013;5(Suppl 4): S407-S412.

4. Krenn L, Kopp B. Bufadienolides from animal and plant sources. Phytochemistry. 1998;48(1):1-29.

5. Qi F, Li A, Inagaki Y, et al. Antitumor activity of extracts and compounds from the skin of the toad Bufo bufo gargarizans Cantor. Int Immunopharmacol. 2011;11(3):342-349.

6. Lu CX, Nan KJ, Lei Y. Agents from amphibians with anticancer properties. Anticancer Drugs. 2008;19(10):931-939.

7. Cui X, Inagaki Y, Xu H, et al. Anti-hepatitis B virus activities of cinobufacini and its active components bufalin and cinobufagin in HepG2.2.15 cells. Biol Pharm Bull. 2010;33(10):1728-1732.

8. Kang XH, Zhang JH, Zhang QQ, et al. Degradation of Mcl-1 through GSK-3 $\beta$ Activation Regulates Apoptosis Induced by Bufalin in NonSmall Cell Lung Cancer H1975 Cells. Cell Physiol Biochem. 2017; 41(5):2067-2076.

9. Miao Q, Bi LL, Li X, et al. Anticancer effects of bufalin on human hepatocellular carcinoma HepG2 cells: roles of apoptosis and autophagy. Int J Mol Sci. 2013;14(1):1370-1382.

10. Xie CM, Chan WY, Yu S, Zhao J, Cheng CH. Bufalin induces autophagy-mediated cell death in human colon cancer cells through reactive oxygen species generation and JNK activation. Free Radic Biol Med. 2011;51(7):1365-1375.

11. Amano Y, Cho Y, Matsunawa M, Komiyama K, Makishima M. Increased nuclear expression and transactivation of vitamin $\mathrm{D}$ receptor by the cardiotonic steroid bufalin in human myeloid leukemia cells. J Steroid Biochem Mol Biol. 2009;114(3-5):144-151.

12. Li D, Qu X, Hou K, et al. PI3K/Akt is involved in bufalin-induced apoptosis in gastric cancer cells. Anticancer Drugs. 2009;20(1):59-64.

13. Shen S, Zhang Y, Wang Z, Liu R, Gong X. Bufalin induces the interplay between apoptosis and autophagy in glioma cells through endoplasmic reticulum stress. Int J Biol Sci. 2014;10(2):212-224.

14. Tsai SC, Yang JS, Peng SF, et al. Bufalin increases sensitivity to AKT/ mTOR-induced autophagic cell death in SK-HEP-1 human hepatocellular carcinoma cells. Int J Oncol. 2012;41(4):1431-1442.
15. Chen YY, Lu HF, Hsu SC, et al. Bufalin inhibits migration and invasion in human hepatocellular carcinoma SK-Hep1 cells through the inhibitions of NF-kB and matrix metalloproteinase-2/-9-signaling pathways. Environ Toxicol. 2015;30(1):74-82.

16. Hu Q, Liang B, Sun Y, et al. Preparation of bufalin-loaded pluronic polyetherimide nanoparticles, cellular uptake, distribution, and effect on colorectal cancer. Int J Nanomedicine. 2014;9(1):4035-4041.

17. Kim SS, Rait A, Rubab F, et al. The clinical potential of targeted nanomedicine: delivering to cancer stem-like cells. Mol Ther. 2014;22(2):278-291.

18. Li M, Zhang W, Wang B, Gao Y, Song Z, Zheng QC. Ligand-based targeted therapy: a novel strategy for hepatocellular carcinoma. Int $J$ Nanomedicine. 2016;11:5645-5669.

19. Zhao R, Matherly LH, Goldman ID. Membrane transporters and folate homeostasis: intestinal absorption and transport into systemic compartments and tissues. Expert Rev Mol Med. 2009;11:e4.

20. O'Shannessy DJ, Yu G, Smale R, et al. Folate receptor alpha expression in lung cancer: diagnostic and prognostic significance. Oncotarget. 2012; 3(4):414-425.

21. Assaraf YG, Leamon CP, Reddy JA. The folate receptor as a rational therapeutic target for personalized cancer treatment. Drug Resist Updat. 2014;17(4-6):89-95.

22. Siafaka P, Betsiou M, Tsolou A, et al. Synthesis of folate-pegylated polyester nanoparticles encapsulating ixabepilone for targeting folate receptor overexpressing breast cancer cells. J Mater Sci Mater Med. 2015;26(12):275.

23. Daniels TR, Bernabeu E, Rodríguez JA, et al. The transferrin receptor and the targeted delivery of therapeutic agents against cancer. Biochim Biophys Acta. 2012;1820(3):291-317.

24. Koshkaryev A, Piroyan A, Torchilin VP. Increased apoptosis in cancer cells in vitro and in vivo by ceramides in transferrin-modified liposomes. Cancer Biol Ther. 2012;13(1):50-60.

25. Sapra P, Allen TM. Improved outcome when B-cell lymphoma is treated with combinations of immunoliposomal anticancer drugs targeted to both the CD19 and CD20 epitopes. Clin Cancer Res. 2004;10(7): 2530-2537.

26. Hare JI, Moase EH, Allen TM. Targeting combinations of liposomal drugs to both tumor vasculature cells and tumor cells for the treatment of HER2-positive breast cancer. J Drug Target. 2013;21(1): $87-96$.

27. Sriraman SK, Salzano G, Sarisozen C, Torchilin V. Anti-cancer activity of doxorubicin-loaded liposomes co-modified with transferrin and folic acid. Eur J Pharm Biopharm. 2016;105:40-49.

28. Gao JQ, Lv Q, Li LM, et al. Glioma targeting and blood-brain barrier penetration by dual-targeting doxorubincin liposomes. Biomaterials. 2013;34(22):5628-5639.

29. Kolhatkar R, Lote A, Khambati H. Active tumor targeting of nanomaterials using folic acid, transferrin and integrin receptors. Curr Drug Discov Technol. 2011;8(3):197-206. 
30. Yuan F, Dellian M, Fukumura D, et al. Vascular permeability in a human tumor xenograft: molecular size dependence and cutoff size. Cancer Res. 1995;55(17):3752-3756.

31. Sahay G, Alakhova DY, Kabanov AV. Endocytosis of nanomedicines. J Control Release. 2010;145(3):182-195.
32. Paulos CM, Reddy JA, Leamon CP, Turk MJ, Low PS. Ligand binding and kinetics of folate receptor recycling in vivo: impact on receptormediated drug delivery. Mol Pharmacol. 2004;66(6):1406-1414.

\section{Publish your work in this journal}

The International Journal of Nanomedicine is an international, peerreviewed journal focusing on the application of nanotechnology in diagnostics, therapeutics, and drug delivery systems throughout the biomedical field. This journal is indexed on PubMed Central, MedLine, CAS, SciSearch ${ }^{\circledR}$, Current Contents ${ }^{\circledR} /$ Clinical Medicine,
Journal Citation Reports/Science Edition, EMBase, Scopus and the Elsevier Bibliographic databases. The manuscript management system is completely online and includes a very quick and fair peer-review system, which is all easy to use. Visit http://www.dovepress.com/ testimonials.php to read real quotes from published authors. 